\title{
Valor económico agregado en las empresas del sector industrial manufacturero de Cúcuta periodo 2008-2012
}

\author{
Economic value added in manufacturing companies in the industrial sector of period \\ 2008-2012 Cúcuta
}
Valor económico agregado nas empresas do setor industrial manufatureiro de Cúcuta período 2008-2012

\author{
Jhon Antuny Pabón-León ${ }^{1}$, Liliana Marcela Bastos-Osorio², Johanna Milena Mogrovejo-Andrade ${ }^{3}$
}

Forma de citar: J. A. Pabón-León, L. M. Bastos-Osorio, J. M. Mogrovejo-Andrade. "Valor económico agregado en las empresas del sector industrial manufacturero de Cúcuta periodo 2008-2012", Respuestas, vol. 20, no. 2, pp. 54-72, 2015.

Recibido:

Noviembre 8 de 2014

Aceptado:

Abril 20 de 2015

\section{4}

\section{Resumen}

Antecedentes: El sector industrial manufacturero en Cúcuta se considera importante por la relevancia que tiene este sector de la economía ya sea por la demanda de productos y servicios, la generación de empleo y el impacto económico que genera en el resto de las actividades económicas en la ciudad. Objetivo: El presente artículo deriva de una investigación sobre la creación de valor en el sector industrial manufacturero de Cúcuta Departamento Norte de Santander Colombia en el periodo 2008-2012, se pretendió describir cómo se comportó este indicador en el sector. Métodos: El trabajo se desarrolló con un enfoque cuantitativo, el alcance de la investigación fue descriptivo con un diseño no experimental. Para ello se efectuó un análisis de tendencias de los indicadores financieros relacionados con los de creación de valor con base en información contable. Resultados: En esta investigación se encontró que no se crea valor en el sector estudiado ni en ninguno de los subsectores que lo conforman en los cinco años de estudio; esto obedece a la disminución de la utilidad operacional y el aumento de los inventarios aunado a que el costo de capital es alto en comparación con el rendimiento del patrimonio. Conclusiones: En la ciudad existe poca vocación industrial, el sector industrial manufacturero en el PIB ha venido perdiendo participación año a año lo que afecta a la economía de la región en sus indicadores sociales y económicos.

${ }^{2}$ Maestría en Gerencia de Empresas lilianamarcelabo@ufps.edu.co ORCID: 0000-0001-6315-4224 Universidad Francisco de Paula Santander Cúcuta-Colombia

${ }^{3}$ Maestría en Gerencia de Empresas johannamilenama@ufps.edu.co ORCID:0000-0002-9694-3382 Universidad Francisco de Paula Santander Cúcuta-Colombia
Palabras clave: Costo de Capital (Ke), Costo Promedio Ponderado de Capital, Cúcuta, Sector Industrial Manufacturero, Valor Económico Agregado (EVA).

\section{Abstract}

Background: The manufacturing industry in Cúcuta is considered important because of the relevance of this sector of the economy either due to demand for products and services, employment generation and economic impact on different other economic activities on the city. Objective: This paper makes part of a research on value creation in the manufacturing sector of Cúcuta Colombia Norte de Santander Department in 2008-2012. It is intended to describe the behavior of this indicator in the sector. Methods: The study 
was developed with a quantitative approach; the scope of the research was descriptive with a non- experimental design. For this, a trend analysis of financial indicators related to value creation based on accounting information was made. Results: This research found that no value is created in the sector or in any of the subsectors studied during five years; this is due to the decrease in operating income and rising inventories coupled with the fact that cost of capital is high compared to the performance of equity. Conclusions: There is low industrial vocation in the city, less contribution of the sector on the GDP year after year, hence regional economy has been affected in both social and economic indicators.

Keywords: Cost of Capital(Ke), Weighted Average Cost of Capital, Cúcuta, Manufacturing Industrial Sector, Economic Value Added (EVA).

\section{Resumo}

Antecedentes: O sector industrial manufatureiro em Cúcuta se considera importante pela relevância que tem este sector da economia, já seja pela demanda de produtos e serviços, a geração de emprego e o impacto económico que gera nas outras atividades económicas da cidade. Objetivo: O presente artigo deriva de uma pesquisa sobre a criação de valor no setor industrial manufatureiro de Cúcuta, Departamento de Norte de Santander, na Colômbia no período 2008-2012. Pretendeu-se descrever como se comportou este indicador no setor. Métodos: $\mathrm{O}$ trabalho se desenvolveu com um enfoque quantitativo, o alcance da pesquisa foi descritivo com um delineamento não experimental. Para isto, efetuou-se uma análise de tendências dos indicadores financeiros relacionados com os de criação de valor com base em informação contável. Resultados: Nesta pesquisa se encontrou que não se cria valor no setor estudado, nem em nenhum dos subsetores que o conformam nos cinco anos de estudo; isto obedece à diminuição da utilidade operacional e o aumento dos inventários somado a que o custo de capital é alto em comparação com o rendimento do patrimônio. Conclusões: Na cidade há pouca vocação industrial, o setor industrial no PIB tem vindo a perder quota a cada ano o que tem afetado a economia da região nos seus indicadores sociais e econômicos.

Palavras-chave: Custo de Capital (Ke), Custo Médio Ponderado de Capital, Cúcuta, Setor Industrial Manufatureiro, Valor Económico Agregado (VEA).

\section{Introducción}

El artículo es el resultado de una investigación previa sobre el Valor Económico Agregado (EVA) en el sector industrial manufacturero de la ciudad de Cúcuta Departamento Norte de Santander Colombia y avance de una investigación de los factores de competitividad en el mismo sector. El EVA es un concepto que en [1] se define como la diferencia entre la utilidad operativa que una empresa obtiene y la mínima que debería obtener, señala también que puede entenderse como el remanente que generan los activos netos de operación cuando producen una rentabilidad superior al costo de capital.
El sector industrial manufacturero en un país es un elemento clave en su economía, clasificado como el sector secundario es un importante multiplicador de las actividades relacionadas, ya sea como demandante de recursos y mano de obra o por los efectos inducidos en la economía. A nivel mundial la participación del sector industrial manufacturero en el Producto Interno Bruto (PIB) alcanza el $30,7 \%$ lo cual es un reflejo de la importancia de este sector, mientras en Colombia ronda el $37,8 \%$ según [2]. La importancia del sector dentro de la economía del país conlleva a su vez la necesidad de evaluar el desempeño de las empresas, en este caso se propone la
ISSN $0122-820 \mathrm{X}$

E-ISSN 2422-5053

PP: 54-72 
No. 2

Julio - Diciembre 2015 ISSN 0122-820X E-ISSN 2422-5053

PP: 54-72 evaluación del desempeño financiero del sector industrial manufacturero de Cúcuta en el periodo 2008-2012apoyándose en el análisis del indicador Valor Económico Agregado (EVA).

La evaluación del desempeño del indicador Valor Económico Agregado (EVA) de las empresas del sector industrial manufacturero en Cúcuta se considera importante por la relevancia que tiene este sector de la economía ya sea por la demanda de productos y servicios, la generación de empleo y el impacto económico que genera en el resto de las actividades económicas en la ciudad.

La investigación se realiza por el interés de conocer el desempeño financiero de las empresas del sector industrial manufacturero de la ciudad de Cúcuta apoyándose en el análisis del indicador Valor Económico Agregado (EVA) y analizar cómo se ha comportado este indicador ya que no se encuentra información al respecto. Por otra parte, establecer indicadores que apoyen la gestión financiera en las empresas es un interés académico. En el ámbito profesional, como Docente, el interés versó en conocer el contexto económico y financiero en el sector en estudio, la investigación permite conocer aspectos internos que pudieran relacionarse con los resultados obtenidos por el sector en los últimos años. Con esto se busca generar algún tipo de conocimiento sobre la realidad de las empresas para coadyuvar a la gestión del desempeño empresarial, buscando minimizar el impacto económico en la ciudad por la disminución de la actividad manufacturera aunado a las repercusiones sociales que esto conlleva.

El EVA fue propuesto como una medida de desempeño que reemplaza medidas tradicionales como la utilidad del ejercicio o el efectivo.
Para [3]:

El EVA podría definirse como el importe que queda una vez se han deducido de los ingresos la totalidad de los gastos, incluidos el costo de oportunidad y los impuestos. Por tanto, el EVA considera la productividad de todos los factores utilizados para desarrollar la actividad empresarial. En otras palabras, el EVA es lo que queda una vez se han atenido todos los gastos $\mathrm{y}$ satisfecho una rentabilidad mínima esperada por parte de los accionistas.

\section{Mientras que en [4]:}

El EVA trata de medir el valor que agrega un proyecto a la firma o el valor que genera la firma en un determinado período. Tiene en cuenta que esa generación de valor debe resultar después de que se ha recuperado lo correspondiente a la inversión y a la remuneración que deben recibir los que prestan el dinero (intereses) y los que aportan el capital (rendimiento de los accionistas).

De acuerdo a [5] la Organización de las Naciones Unidas para el Desarrollo Industrial manufacturero (ONUDI) analiza la influencia de la industrialización en el cambio de las economías y la evolución de este proceso en los últimos años. En términos de Valor Agregado Industrial manufacturero (VAM) destaca el caso de Latinoamérica, cuya aportación al VAM de los países en vías de industrialización pasó del 35\% en 1992 al $17 \%$ en 2012. En el caso de Colombia según las cifras aportadas por [6] la producción industrial manufacturero había caído 2,4\% de enero a octubre y adicionalmente el valor agregado de la industria se contrajo $0,7 \%$, siendo la única actividad que registró caídas anuales en los últimos tres trimestres del año.

Por su parte en el artículo "Crece preocupación por desempleo en Cúcuta" (2012, marzo 01) se indica que los gremios económicos de la ciudad le reclamaron al Ministro de Trabajo 
una verdadera política de empleo local en Cúcuta ante la alta cifra de desocupados que tiene hoy la capital norte santandereana, y que la ubican en la tercera ciudad con más desocupación. Se reseña también que esa ciudad, alcanzó el 16.1\%, viéndose reflejado en la pérdida de más de sesenta mil trabajos en el último trimestre, encendiéndose las alarmas por la grave crisis social y económica que tiene el oriente del país [7]. De 48.269 personas que estuvieron vinculadas al sector manufacturero entre diciembre de 2012 y febrero de 2013 se pasó a 41.789 empleados entre diciembre de 2013 y febrero de 2014 [6].

Ante la situación planteada surgió el interés por dar respuesta a la siguiente interrogante ¿Crearon valor las empresas del sector industrial manufacturero de Cúcuta en el periodo 2008-2012?, para ello se plantea como hipótesis de trabajo: "Las empresas del sector industrial manufacturero de Cúcuta en el periodo 2008-2012 crearon valor".

Por lo anteriormente expuesto en la investigación se plantea como objetivo general determinar el Valor Económico Agregado (EVA) en las empresas del sector industrial manufacturero de Cúcuta en el periodo 20082012.Para ello se plantea como objetivos específicos: caracterizar el sector industrial manufacturero de la ciudad de Cúcuta, cuantificar el costo promedio ponderado de capital en el sector en estudio, identificar el comportamiento de las variables financieras requeridas para calcular el EVA en el sector.

El trabajo se desarrolló con un enfoque cuantitativo, como un estudio descriptivo de corte transversal no experimental. Se estructuró de la siguiente forma: Introducción. - Materiales y Métodos. Aquí se presenta el marco teórico donde se desarrolla el marco conceptual para el cálculo del EVA (Valor Económico Agregado, por sus siglas en inglés), el marco de referencia, en el cual se presentan estudios relacionados con el tema en el ámbito nacional e internacional y la metodología seguida para el análisis de la información. - Resultados y Análisis. Se determina el EVA de los sub sectores objeto de estudio. -Conclusiones del trabajo de investigación y se dejan planteadas algunas inquietudes que podrían servir de base para futuros trabajos.

\section{Marco teórico y marco de referencia}

\subsection{EI Valor Económico Agregado}

En [8] se documenta que el concepto del EVA es una variación o modificación de lo que tradicionalmente se conocía como "Ingreso o beneficio residual", es decir, el resultado que se obtenía al restar a la utilidad operacional los costos del capital. Agrega que "La idea del beneficio residual apareció a principios del siglo XX, en la literatura contable. Church lo introdujo en 1917 y luego, en 1924, Scovell citó el beneficio residual; ya para 1960 aparece dicho concepto y se contempla en la literatura de la contabilidad gerencial".

Sobre el asunto en [8] hace referencia a la antigüedad del concepto en el inicio del siglo XX. Continúa señalando que "Diez años más tarde, para la década de los 70, los académicos finlandeses discuten sobre dicho tema y en 1975 Virtanen lo define como un retorno sobre inversión, para la toma de decisiones gerenciales". En este apartado menciona como se retoma el concepto en la década de los setenta.

Si bien es cierto que el objetivo financiero primario de cualquier negocio, es el maximizar las riquezas de sus accionistas. Según [9] "una empresa que crea valor no solo beneficia a sus accionistas, sino también el valor de todos los derechos de las otras partes involucradas, mientras que en cambio, todas las partes se resienten cuando la dirección fracasa en la creación de valor para el accionista."

Así mismo [1, p.129] indicó que la medida del valor agregado del período se denomina Ganancia Económica o Ingreso Residual. Sin embargo el nombre más utilizado para referir
Julio - Diciembre 2015 ISSN 0122-820X E-ISSN 2422-5053 PP: 54-72 
No. 2

Julio - Diciembre 2015 ISSN 0122-820X E-ISSN 2422-5053

PP: 54-72 este fenómeno es EVA, Economic Value Added, que es una marca registrada de la firma consultora de los Estados Unidos, Stern Stewart \& Co.

Dentro de ese marco [10] señala que el EVA es una medida popular que usan muchas empresas para determinar si una inversión (propuesta o existente) contribuye positivamente a la riqueza de los propietarios e indica que la aplicación del EVA requiere el uso del costo de capital promedio ponderado (CPPC).

De acuerdo a la metodología recomendada por [11] citado por [12] para determinar el valor económico agregado (EVA) del sector se efectúa el cálculo de una utilidad económica en un periodo t; al determinar la utilidad residual, se le deduce a la utilidad operacional después de impuestos (UODI), el cargo de capital o costo de los recursos invertidos en la operación. Si la diferencia es positiva es porque la empresa crea valor, si es negativa es porque está destruyendo valor, y si es cero es porque mantiene un equilibrio económico.

Otro indicador que permiten evaluar y controlar el EVA es la razón EVA/ANO, según [13] "con la razón EVA/ANO se determina el valor económico agregado generado por cada peso de inversión en activo neto operacional".

\subsection{Costo Promedio Ponderado de Capital (CPPC)}

Vinculado al concepto [1, p.247] lo define como el costo promedio ponderado de todos proporción entre la deuda y el patrimonio que se utiliza para financiar activos, se conoce con el nombre de estructura de capital o estructura financiera y es la que se utiliza en su cálculo, con la siguiente formula:

$$
C C P C=K d(1-\mathrm{T}) r D+\operatorname{Ker} P
$$

CPPC $(\mathrm{Ko})=$ (Deudas de terceros $/$ Total de financiamientos) $x$ (costo de la deuda) $x$ (1tasa de impuestos)+ (Capital Propio / Total de financiamientos) $x$ (costo del capital propio)

Donde:rP: Razón patrimonial

Ke: Costo del Patrimonio

rD: Razón de endeudamiento

Kd: Costo promedio de la deuda

(1-T): Beneficio tributario, donde T representa la tasa de Impuestos.

\subsection{CAPM (Capital Assets Pricing Model)}

Indicó así mismo [1, p.248] que el Costo del Patrimonio "...no es una cifra que se establece en forma caprichosa sino el resultado de un ejercicio que considera la rentabilidad que ganan las empresas del sector, y el riesgo país. Estos elementos son considerados por el que es tal vez el método más utilizado para calcular el costo del patrimonio, como es el modelo del CAPM (Capital Assets Pricing Model).

Partiendo de los supuestos anteriores [14] sugiere que el método ha llegado a ser un concepto que tiene amplias aplicaciones, entre ellas se puede mencionar que es útil para determinar el costo del capital patrimonial y para evaluar los activos riesgosos.

Siguiendo a [15] con el modelo CAPM se estima que el costo del capital propio $\mathrm{Ke}$ es igual a la tasa libre de riesgo Rf, más el riesgo sistemático de la empresa $\beta$ multiplicado por la prima de riesgo de mercado $R m-R f$. Por lo tanto, $\mathrm{Ke}$ es equivalente a:

$K e=R f+\beta(R m-R f)$

Para tal efecto de acuerdo a los autores, se asume que el costo de oportunidad de los propietarios de las empresas de un sector de un país emergente sería muy similar al de un país desarrollado referente, con mercados bursátiles más competitivos y eficientes, más una prima por riesgo país RP, por tener la inversión en un país emergente dado que esto evitaría las restricciones teóricas y técnicas para el cálculo del beta en países emergentes que, como sucede en Colombia, mantienen un 
mercado de renta variable pequeño, ilíquido y concentrado.

De esta forma, el costo de oportunidad de las empresas de un sector del país emergente sería igual a:

$K e=R f+\beta(R m-R f)+R P$

Más los ajustes correspondientes por inflación [1, p.248].

Al referirse al cálculo del CAPM en mercados emergentes en [16, p.290] se recomienda el siguiente procedimiento:

a) Seleccionar un grupo de empresas similares a la empresa del país emergente en un país desarrollado (generalmente se toma a los Estados Unidos).

b) Obtener la betas de dichas empresas (betas apalancadas).

c) Desapalancar las betas.

d) Calcular el promedio de las betas desapalancadas.

e) Calcular el costo de capital con el CAPM.

f) Determinar el costo de capital en términos reales.

g) Sumar el riesgo país.

h) Incorporar la inflación nacional en la tasa de descuento.

Señala [16, p.292] que "el resultado que se obtiene con este procedimiento debe tratarse con precaución y siempre debe ser objeto de un riguroso análisis de sensibilidad".

\subsection{EVA y Los Procesos Administrativos}

Dentro de este marco, en opinión de [17] hay autores que indican, que adoptar un pensamientoorientadoacrearvaloreidentificar los elementos claves lleva a la empresa sólo hasta la mitad del camino. Los gerentes deben establecer procesos que involucren a todos los empleados en la necesidad de crear valor. Cuatro procesos principales rigen la adopción de la GBV: primero, desarrollar estrategias para maximizar el valor; segundo, traducir la estrategia en metas de corto y largo plazo que se enfoquen en los principales inductores de valor; tercero, desarrollar planes de acción y presupuestos enfocados al cumplimiento de las metas de corto y mediano plazo; y cuarto, introducir sistemas de medición de resultados y esquemas de compensación con el fin de monitorear e incentivar a los empleados para que cumplan las metas establecidas. Estos cuatro procesos deben estar conectados en los niveles corporativos, de unidades de negocio y funcionales. Es evidente que las estrategias y los resultados operativos deben ser consistentes a todo lo largo y ancho de las organizaciones para que el objetivo de crear valor se cumpla.

En Colombia se han efectuado diversos estudios sobre el EVA:

Se encontró la investigación "Impacto del cargo de capital sobre el valor de las empresas innovadoras de confecciones de Cali" [15], los autores siguiendo los lineamientos teóricos de la gestión basada en el valor y, como metodología, el análisis del valor económico agregado en el periodo 20022007. Se encuentra que el sector destruye valor debido a que el incremento del activo no conduce a un crecimiento semejante de la utilidad operacional después de impuestos, pero sí al aumento del cargo de capital, pese a la reducción del riesgo país. Se generan nuevos interrogantes en cuanto si la inversión en innovación va a mejorar la productividad en un futuro y si el costo de capital va a continuar descendiendo.

Vinculados al concepto en [18] se analiza la "Creación de valor en la industria textil". Como resultado del modelo planteado se llega a la conclusión de que ninguna de ellas mantiene una tendencia marcada y que los diferentes métodos no son afines entre sí en los mismos periodos; sin embargo, se
Julio - Diciembre 2015 ISSN 0122-820X E-ISSN 2422-5053 PP: 54-72 
No. 2

Julio - Diciembre 2015 ISSN 0122-820X E-ISSN 2422-5053

PP: 54-72 orienta mejor la toma decisiones mediante la interpretación de los resultados, en lugar de depender exclusivamente de los registros financieros.

Según [19] en "Creación de Valor de las Empresas Colombianas durante el Período 2000-2005", se muestra el análisis sobre la generación de valor en la economía colombiana en 60 sectores. Indican que la información fue tomada de la base de datos Análisis Financiero y de Negocios (AFINE).

"Medición del valor económico agregado EVA de las empresas de Caldas en el periodo 2000-2008" [20]. La investigación está basada en el reporte de información de los estados financieros consignados de forma continua por las empresas de Caldas durante el periodo 2000-2008 ante la Superintendencia de Sociedades que permitió calcular y obtener la variación de dicho índice.

"Las empresas de alimentos de Manizales generan ganancias pero destruyen valor económico agregado (EVA)" [21]. Dicha investigación tiene en cuenta a 19 empresas de la ciudad. Da cuenta de cómo las empresas estudiadas de Manizales obtienen ganancias pero destruyen valor económico agregado, caso que, corregido, permitiría a las empresas ser más eficientes, efectivas y obtener mayores ganancias.

El estudio “Análisis del desempeño financiero de empresas innovadoras del sector alimentos y bebidas en Colombia" [13]. La investigación se efectúa para el período 2000-2008, y se utilizan indicadores financieros con base en información contable y de valor económico agregado. Se encontró que las empresas innovadoras solo crean valor hasta el 2003, a partir de 2004 comienza a destruirlo, sin mostrar tendencias de recuperación o deterioro. Esto obedece principalmente al comportamiento de la eficiencia en el uso de los activos.

Mientras que en ¿Los medios de comunicación en Colombia son una industria creadora de valor? [12]. Se encontró que dicho sector solo crea valor en uno de los seis años de estudio; esto obedece a que el sector presenta una alta dependencia en la eficacia con que se han gestionado los gastos de administración y del ajuste de su estructura de capital para lograr un menor costo de capital.

\section{Materiales y métodos}

El trabajo se desarrolló con un enfoque cuantitativo, basado en la revisión de información tomada de los estados financieros de las empresas del sector industrial manufacturero que reportaron a SIREM [22] en el período de estudio y se apoyó también en informes de Cámara de Comercio de Cúcuta sobre el Producto Interno Bruto (PIB) departamental. Se aplicó el análisis de tendencias de los indicadores financieros relacionados con los de creación de valor con base en información contable para determinar el EVA en las empresas del sector industrial manufacturero de Cúcuta en el periodo 20082012.

La investigación se desarrolló como descriptiva con un diseño no experimental. Se analizaron los estados financieros de las empresas del sector industrial manufacturero de la ciudad de Cúcuta en los años 2008 a 2012.

Con el fin de identificar los subsectores más representativos del sector industrial manufacturero se analizó para cada uno de los años considerados los resultados de la Encuesta Anual Manufacturera (EAM) [23] aplicada por DANE, cuyo objetivo es obtener información básica del sector industrial, que permita el conocimiento de su estructura, características y evolución. La información se consultó en los cuadros "Variables Principales según Áreas Metropolitanas y Grupos Industriales, Total Nacional" y se clasificó la información atendiendo a: personal ocupado, producción bruta y valor agregado. Se escogieron las cinco clases con mayor valor en cada variable, una vez clasificadas 
se le asignó un peso de cinco (por ser cinco clases) a la de mayor valor, cuatro a la de valor inmediato inferior y así sucesivamente, posteriormente se sumaron los pesos.

Una vez identificados los subsectores más representativos del sector industrial manufacturero de la ciudad de Cúcuta se consultó la información financiera. Para identificar la población se consideró como criterio de inclusión que las empresas hayan reportado regularmente en el periodo 20082012.En la investigación se encontró que en promedio veintiséis (26) empresas de las clases industriales seleccionadas reportaron los estados financieros ante el organismo citado.

La información se adaptó para el cálculo de la utilidad operativa después de impuestos (UODI), el Costo Promedio Ponderado de Capital (CPPC) o WACC por sus siglas en inglés y los activos netos de operación (ANO), y así calcular el EVA mediante la expresión:

$E V A=U O D I-((C P P C)(A N O))$

De acuerdo con esta versión del EVA, una compañía destruye valor cuando la UODI de la empresa es menor al CPPC multiplicado por los activos netos de operación (ANO).

Aqui: UODI= Utilidad antes de intereses e impuestos * $(1-t)$

$A N O=$ Activo neto operacional $=$ capital de trabajo neto operacional (KTNO)+ Activo fijo neto Operacional (AFNO)

$\mathrm{CPPC}=$ Costo Promedio Ponderado de Capital $=$ El costo promedio ponderado del capital se obtuvo de la suma de los costos de financiación de todos los recursos, los obtenidos de fuentes externas (Kd)y los recursos propios $(\mathrm{Ke})$. Los recursos con la banca $(\mathrm{Kd})$ se consideraron después de impuestos $\mathrm{Kd}$ (1-t) multiplicado por el porcentaje de endeudamiento y los recursos propios de los accionistas $(\mathrm{Ke})$ multiplicado por el porcentaje de recursos propios en la estructura de financiamiento, así:
$C P P C=$ (Deudas deterceros /Total definanciamientos) $x$ (costo de la deuda) x (1-tasa de impuestos) + (Capital Propio / Total de financiamientos) $x$ (costo del capital propio

Para determinarel costo de los recursos propios se utilizó el Modelo de Costos de Activos de Capital (CAPM).Dada la complejidad para determinar en empresas que no cotizan en la Bolsa de Valores, se siguió la metodología utilizada por [15, p.89-90] Dado que $\beta$ del modelo CAPM representa el riesgo total de la empresa $\beta \mathrm{L}$, que incluye el riesgo sistemático operativo y financiero, se considera que habría afinidad entre la empresa emergente y el país desarrollado en el riesgo operativo $\beta \mathrm{u}$.

Entonces, se hace necesario calcularlo así:

$\beta u=\beta \mathrm{L} /[1+(1-\mathrm{t}) D / S]$

Posteriormente se calculó la $\beta \mathrm{L}$ de la empresa del país emergente con el objeto de considerar la protección fiscal de su propio apalancamiento, empleando:

$\beta L=\beta u *[1+(1-t) D / S]$

Con la beta total $\beta \mathrm{L}$ se determinó Ke de la empresa del país emergente, utilizando la ecuación (3), tomando información de Rf, $\beta \mathrm{L}, \mathrm{Rm}$ y $\mathrm{t}$ del mercado de valores del país desarrollado.

Pero este Ke está expresado en moneda extranjera del país desarrollado; por lo tanto, se debe convertir en moneda local de la siguiente forma:

Ke moneda local $=[(1+$ Ke moneda extranjera $)$

$*(1+$ Devaluación $)]-1$ ISSN 0122-820X ISSN $2422-5053$ PP: 54-72

Si se considera una devaluación en condiciones de paridad cambiaria, se utiliza la siguiente expresión:

Devaluación $=(1+$ inflación interna $) /$

$(1+$ inflación externa $)$

En opinión de [11, p.94] "se puede decir que el EVA depende de tres grandes inductores: 1) 
No. 2

Julio - Diciembre 2015 ISSN 0122-820X E-ISSN 2422-5053

PP: 54-72 la utilidad operacional después de impuestos; 2) el activo neto operacional, y 3) el costo de capital". Con estos inductores se puede determinar el valor del EVA así:

$E V A t=(U A I I t)(1-t)-(A N O t-1)$
$*(C P P C t)$

Si el resultado es positivo es porque se ha creado valor y si es negativo es porque se ha destruido.

\section{Resultados y análisis}

La participación del sector industrial manufacturero del Departamento Norte de Santander en el PIB departamental en el período de estudio se muestra en la Tabla I.

Tabla I. Participación de la industria en el PIB del norte de Santander (\%)

\begin{tabular}{|l|c|c|c|c|c|}
\cline { 3 - 6 } & 2008 & 2009 & 2010 & 2011 & 2012 \\
\hline Participación de la industria en el PIB del Norte de Santander (\%) & 8,7 & 8 & 7,7 & 7,5 & 7,4 \\
\hline Variación (\%) términos nominales & & $-8 \%$ & $-4 \%$ & $-3 \%$ & $-1 \%$ \\
\hline
\end{tabular}

Fuente: Los Autores con datos de la Cámara de Comercio de Cúcuta [24]

Se observó una tendencia decreciente en la contribución del sector en el PIB, la mayor caída se observa en el año 2009 con respecto al año 2008 donde alcanzó el ocho por ciento en términos nominales. En los años 2010 y 2011 la caída en términos nominales estuvo en el orden del uno por ciento y se aceleró en el 2012 al alcanzar el dos por ciento de disminución respecto al año 2011.

El Valor Agregado a la economía entendida como Producción Bruta menos Consumo Intermedio mostró las cifras siguientes. Ver Tabla II.

Tabla II. Comportamiento valor agregado a la economía por el sector industrial manufacturero años 2008-2012

\begin{tabular}{|c|c|c|c|c|}
\hline & $\begin{array}{c}\text { VALOR AGREGADO } \\
\text { A LA ECONOMIA }\end{array}$ & $\begin{array}{c}\text { VALOR AGREGADO } \\
\text { TOTAL NACIONAL }\end{array}$ & $\begin{array}{c}\text { PESO VALOR } \\
\text { AGREGADO CUCUTA } \\
\text { EN EL NACIONAL }\end{array}$ & $\begin{array}{c}\text { VARIACION } \\
\text { INTERANUAL } \\
\text { CUCUTA }\end{array}$ \\
\hline 2008 & $20.875 .716,40$ & $65.014 .561 .489,00$ & $0,03 \%$ & \\
\hline 2009 & $33.205 .548,25$ & $64.669 .332 .622,00$ & $0,05 \%$ & $59,1 \%$ \\
\hline 2010 & $20.616 .874,60$ & $67.802 .873 .941,00$ & $0,03 \%$ & $-37,9 \%$ \\
\hline 2011 & $180.793 .526,20$ & $76.338 .526 .321,00$ & $0,24 \%$ & $776,9 \%$ \\
\hline 2012 & $25.906 .732,60$ & $75.865 .747 .833,00$ & $0,03 \%$ & $-85,7 \%$ \\
\hline & & & & \\
\hline PROMEDIO & $56.279 .679,61$ & $69.938 .208 .441,20$ & $0,08 \%$ & \\
\hline Total & $281.398 .398,05$ & $349.691 .042 .206,00$ & $0,09 \%$ & \\
\hline
\end{tabular}

Fuente: Los Autores con datos de [23]

El valor agregado a la economía no muestra tendencias en el periodo, se observó una fuerte contribución en el año 2011 la cual fue impulsada por la clase industrial 269 (fabricación de productos minerales no metálicos), aunque en el año 2012 retoma el comportamiento promedio evidenciado en los demás años. Se evidencia también un pequeño porcentaje de participación del sector industrial de la ciudad al compararlo con el total nacional representando en promedio el $0,09 \%$.

Al determinar las clases industriales más representativas, se encontró que las divisiones y clases industriales de acuerdo a la Clasificación Industrial Internacional Uniforme rev. 3 adaptada para Colombia fueron los mostrados en la Tabla III. 
Jhon Antuny Pabón-León, Liliana Marcela Bastos-Osorio, Johanna Milena Mogrovejo-Andrade

Tabla III. Divisiones y clases industriales según CIUU rev 3 más representativas

\begin{tabular}{|c|l|c|l|}
\hline División & Descripción & Clase & Descripción \\
\hline 15 & Elaboración de alimentos y bebidas & 154 & $\begin{array}{l}\text { Productos molinería, almidones y derivados de } \\
\text { almidón y alimentos para animales }\end{array}$ \\
\cline { 2 - 4 } & & 159 & Elaboración de bebidas \\
\hline 19 & $\begin{array}{l}\text { Curtido y preparado de cueros, fabricación de } \\
\text { calzado, artículos de viaje }\end{array}$ & 192 & Fabricación de calzado \\
\hline 26 & $\begin{array}{l}\text { Fabricación de productos minerales no } \\
\text { metálicos }\end{array}$ & 269 & Fabricación de productos minerales no metálicos \\
\hline 36 & Fabricación de muebles & 369 & $\begin{array}{l}\text { Otras industrias manufactureras fabricación de } \\
\text { muebles }\end{array}$ \\
\hline
\end{tabular}

Fuente: Los Autores con datos de [23]

Atendiendo a las variables analizadas se determinó la participación por clase industrial el siguiente orden. Ver Tabla IV.

Tabla IV. Clasificación de las clases industriales según participación en la economía de la ciudad

\begin{tabular}{|c|c|l|}
\hline Posición & Clase & Descripción \\
\hline 1 & 269 & Fabricación de productos minerales no metálicos \\
\hline 2 & 369 & Otras industrias manufactureras fabricación de muebles \\
\hline 3 & 159 & Elaboración de bebidas \\
\hline 4 & 154 & $\begin{array}{l}\text { Productos molinería, almidones y derivados de almidón } \\
\text { yalimentos para animales }\end{array}$ \\
\hline 5 & 192 & Fabricación de calzado \\
\hline
\end{tabular}

Fuente: Los Autores con datos de [23]

El resultado consolidado de la clasificación de acuerdo a las variables estudiadas se muestra en la Grafica 1

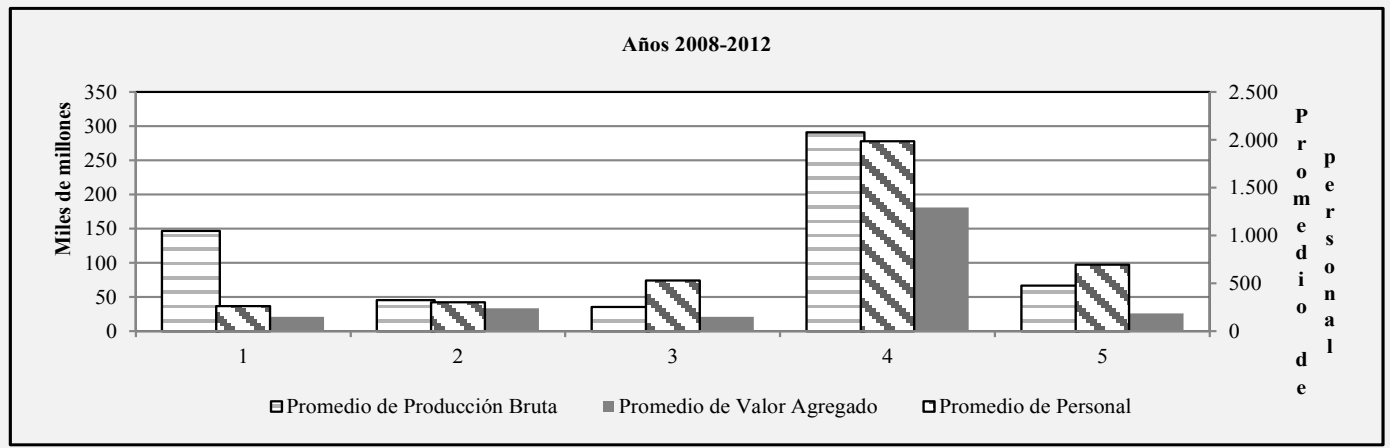

Grafica 1. Clasificación grupos industriales consolidado periodo de estudio Fuente: Los Autores

Se verifica que la clase industrial 269 es la que muestra el mayor nivel de participación en la economía de la ciudad.

En la Grafica 2 y Grafica 3 se presentan los grupos industriales más representativos de acuerdo a las variables analizadas en cada año de estudio.

Se confirma que las cinco clases industriales presentan un comportamiento similar en cada uno de los años estudiados sin presentar cambios considerables en su participación. 
No. 2

Julio - Diciembre 2015 ISSN 0122-820X E-ISSN 2422-5053
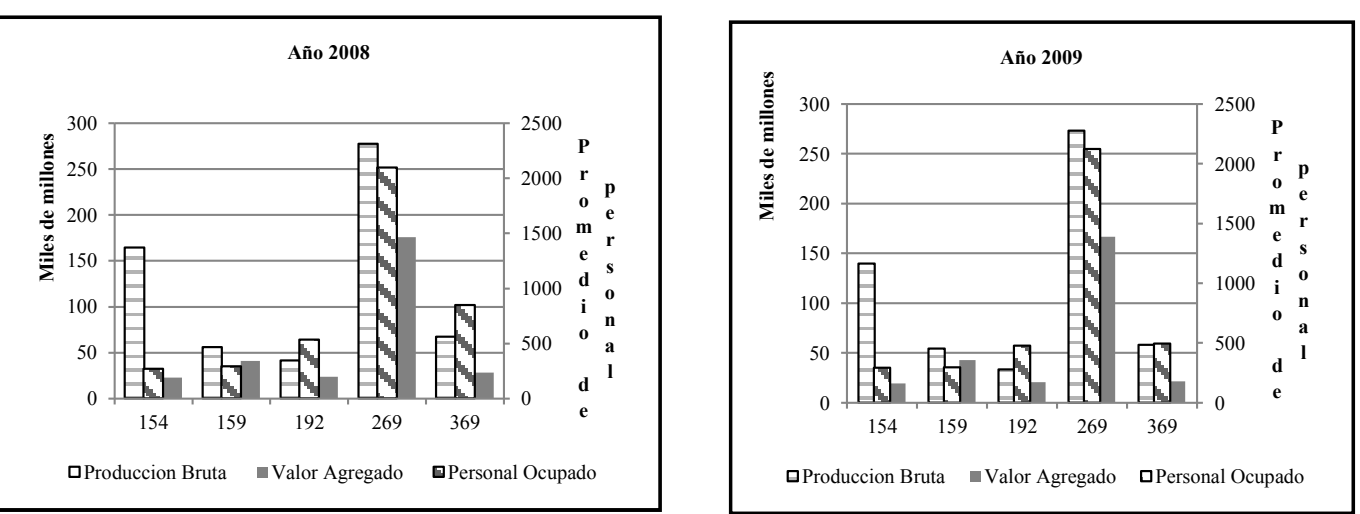

Grafica 2. Clasificación grupos industriales por año de estudio Fuente: Los Autores
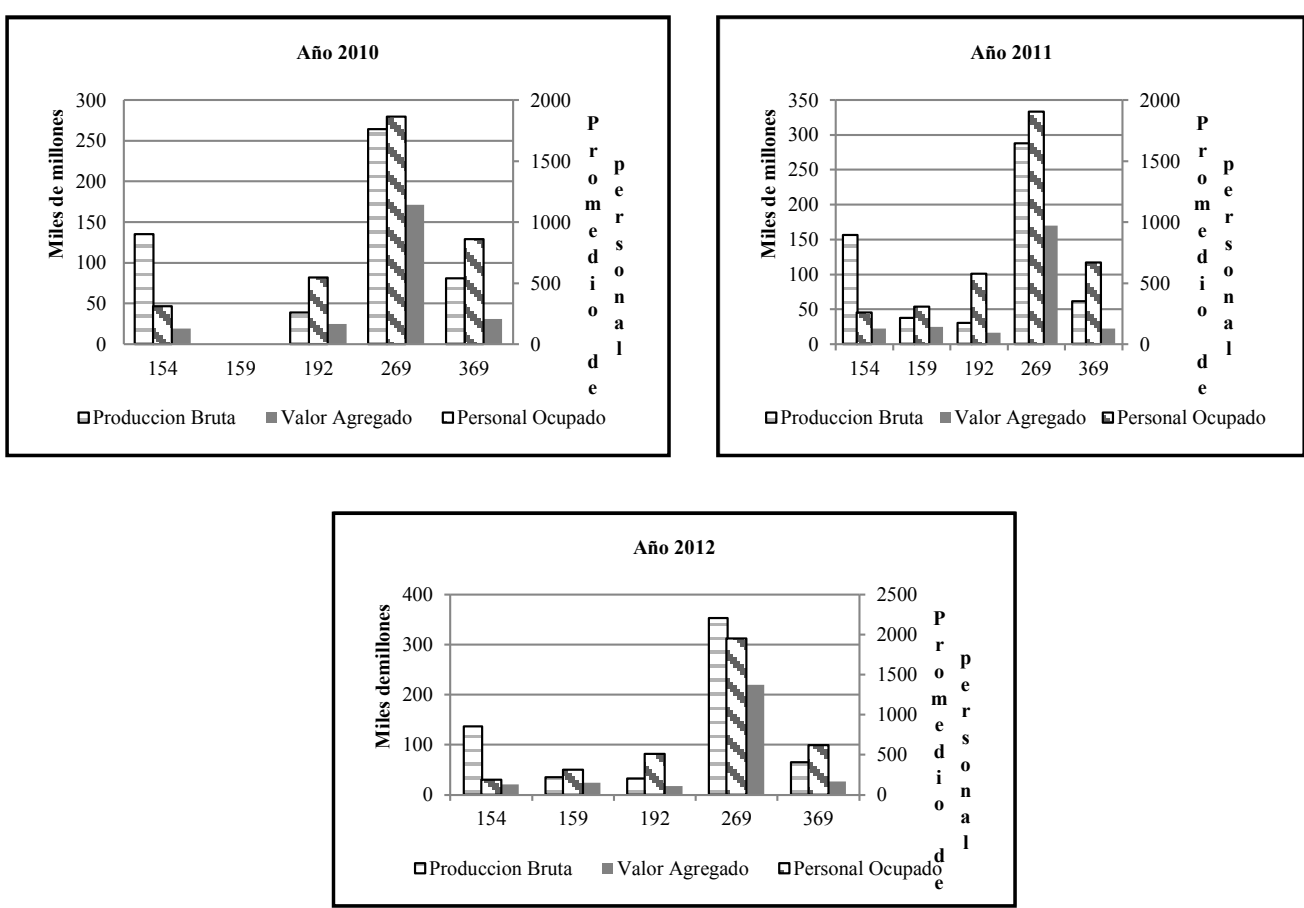

Grafica 3. Clasificación grupos industriales por año de estudio Fuente: Los Autores

En el análisis se determina que las clases industriales 269 (fabricación de productos minerales no metálicos) y la 369 (otras industrias, fabricación de muebles) son las más representativas en cuanto a la ocupación de personal en los años de estudio.

Se muestra a continuación los resultados del EVA para el sector manufacturero y para las clases industriales seleccionadas. Los datos y cálculos se despliegan en los anexos. Ver las Tablas VII, VIII, IX, X y XI.

En la Tabla V se presentan las fuentes de información para aplicar el modelo CAPM. 
Tabla V. Fuentes de datos para aplicación del modelo CAPM

\begin{tabular}{|l|l|}
\hline Datos & Significado y Fuente \\
\hline Rf, & T-Bond rate Damodaran on line \\
$\mathrm{Rm}$ & Beta desapalancada Damodaran on line \\
\hline $\mathrm{Kd}$ & Total Equity Risk Premium Damodaran on line \\
\hline $\mathrm{RP}(\mathrm{EMBI})$ & Banco Mundial \\
\hline Inflación en Colombia & Banco Central de Perú-Estadísticas \\
\hline Inflación en Estados Unidos & México Maxico \\
\hline
\end{tabular}

Fuente: Los Autores con base en [25], [26], [27], [28] y [29].

La Grafica 4 muestra el resultado del EVA y del indicador EVA/ANO en el sector industrial manufacturero de Cúcuta años 2008-2012.
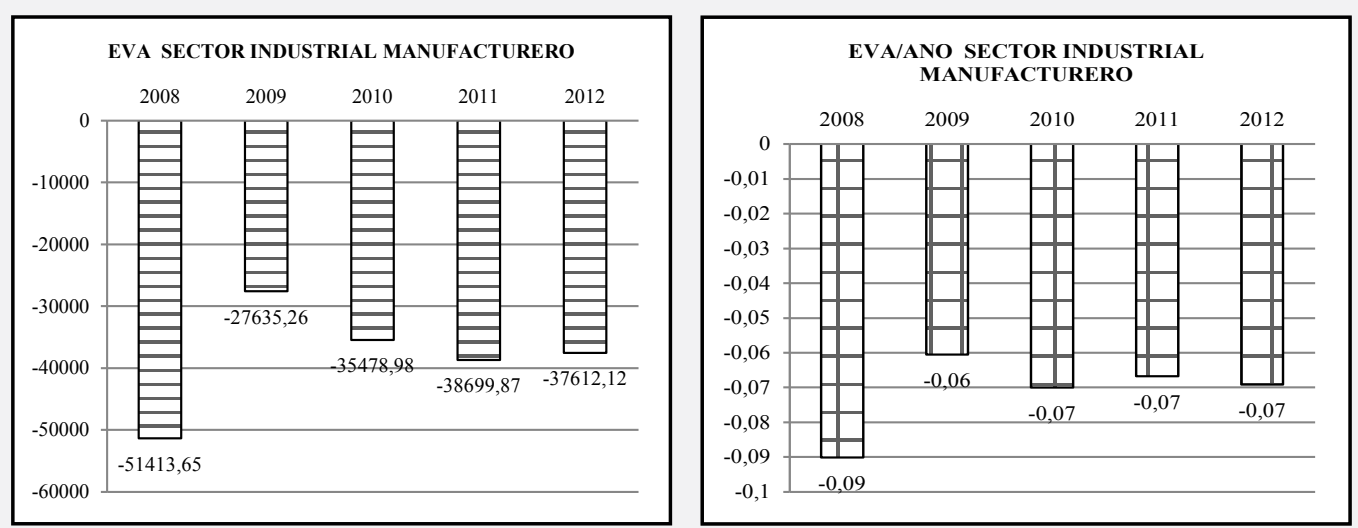

Grafica.4 EVA y relación EVA/ANO del sector manufacturero Fuente: Los autores

Se verifica que se destruye valor en los años del periodo de estudio, se puede observar tres comportamientos diferentes años 2008 -2009; años 2009-2011 y años 2011-2012. Entre el año 2008 y 2009 se mejoró en la creación de valor al pasar de $-51.413,65$ a -27.635,26 millones de pesos, luego entre 2009-2011 desmejora y cae a $-35.478,98$ millones de pesos observándose una disminución en el ritmo entre 2010 y 2011, la tendencia se revierte en el año 2012 al mejorar el indicador a -37.612,12 millones de pesos. La relación EVA/ANO que expresa centavos/ peso invertido en activos muestra dos comportamientos muy definidos. Entre 2008 y 2009 mejora y pasa de - 0,09 a - 0,06 en el año 2009 y entre los años 2010 y 2012 donde permaneció relativamente estable alrededor de $-0,07$.

Los resultados de la clase industrial 269 (fabricación de productos minerales no metálicos) se presentan en la Grafica 5. 
No. 2

Julio - Diciembre 2015 ISSN 0122-820X E-ISSN 2422-5053
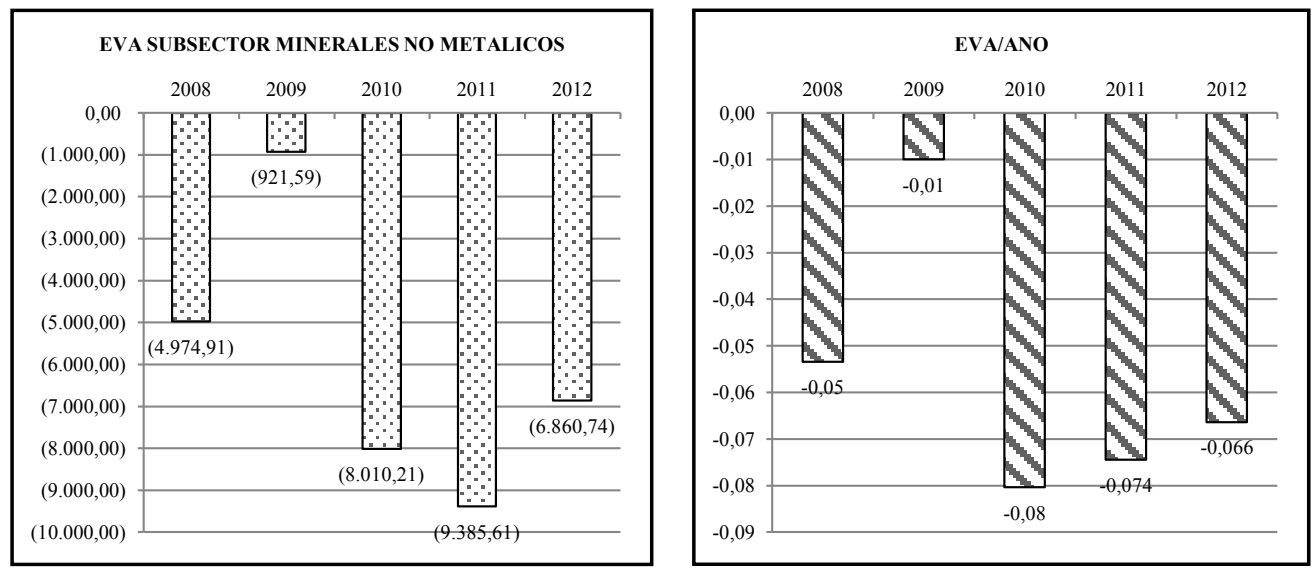

Grafica 5. EVA y relación EVA/ANO clase industrial 269 Fuente: Autores

En el resultado se evidencia que se destruye valor en todo el periodo de estudio, se presentan tres ciclos bastante definidos entre los años 2008-2009, años 2009-2011 y años 2011-2012. Entre el año 2008 y 2009 se observó la mejora del indicador al pasar de -4.974,91 a -921,59 millones de pesos, entre los años 2009 y 2011 desmejoró notablemente y llegó a -9.385,61 millones de pesos y se revirtió para el año 2012 cuando alcanzó -6.860,74 millones de pesos. Por su parte el indicador EVA/ANO evidenció entre los años 2010 y 2012 recuperación paulatina donde la pérdida de centavos por peso invertido en activos pasó de -0,08 a -0,066.

Resultados clase industrial 369 (Otras industrias manufactureras, fabricación de muebles). Los resultados se exponen en la Grafica 6.


Grafica 6. EVA y relación EVA/ANO clase industrial 369 Fuente: Los Autores

El comportamiento del indicador presentó un comportamiento variable año a año, se verificó que en el año 2009 el indicador fue positivo pero en los restantes años destruyó valor. Se identifican igualmente tres ciclos entre 2008 y 2009, entre 2009 y 2010 y entre 2010 y 2012. Entre 2009 y 2010 se observó que el EVA desmejoró notablemente al pasar de $+10,97$ a -218,17. Entre tanto el índice EVA/ANO mostro que la pérdida pasó de cinco centavos a un centavo por peso invertido en activos.

Resultados clase industrial 154 (productos molinería, almidones y derivados de almidón y alimentos para animales). En la Grafica 7 se puede observar el comportamiento. 

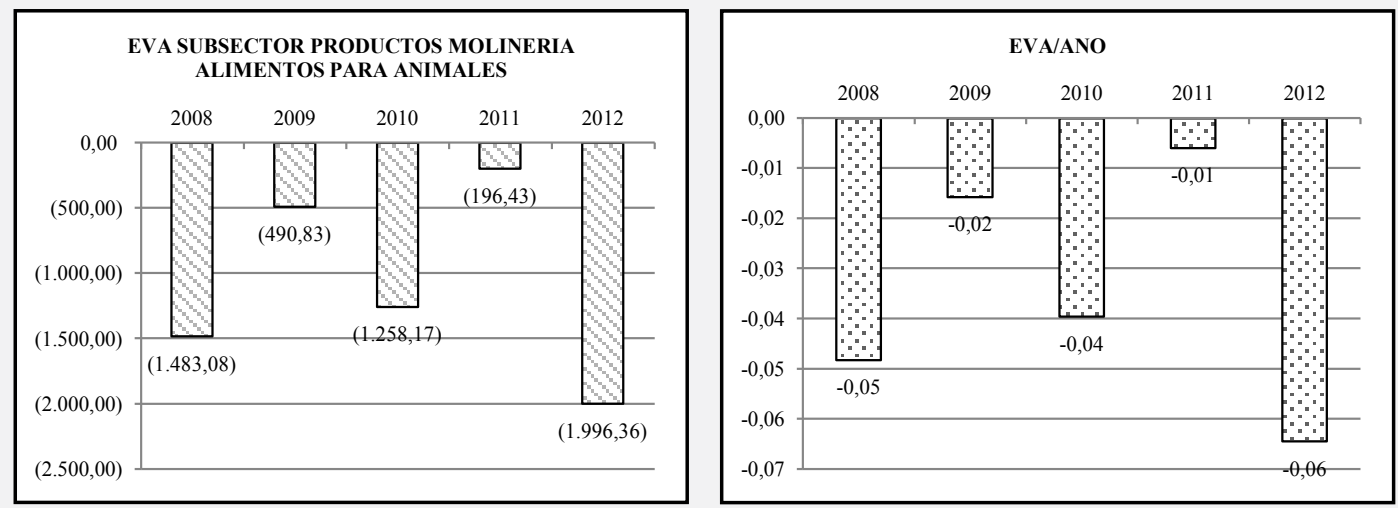

Julio - Diciembre 2015

ISSN 0122-820X

E-ISSN 2422-5053

PP: 54-72

Grafica 7. EVA y relación EVA/ANO clase industrial 154

Fuente: Los Autores

El resultado del EVA revela que se destruyó valor en todos los años del estudio, el comportamiento fue variable año a año y no se observó una tendencia definida. Se determinó que entre el año 2011 y 2012 esta clase industrial presentó una fuerte caída en el indicador al pasar de -196,43 millones de pesos a -1.996,36 millones de pesos en el año 2012. Igual comportamiento variable se observó en el indicador EVA/ANO, llama la atención el resultado de los años 2011 y 2012 en el cual presentó para el año 2012 una pérdida de 0,06 centavos por peso invertido en activos habiendo mostrado un centavo por peso el año anterior. Resultados clase industrial 192 (fabricación de calzado). Ver Grafica 8.
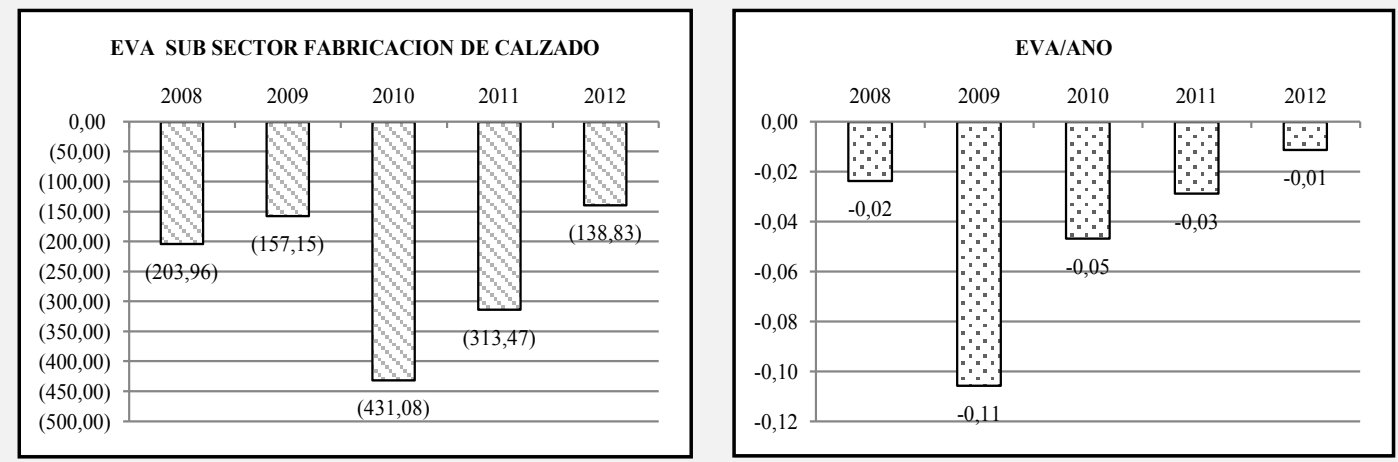

Grafica 8. EVA y relación EVA/ANO clase industrial 192 Fuente: Los Autores

En todos los años el valor del EVA mostró que se destruyó valor. Se verifica que entre los años 2009 y 2010 el indicador desmejoró al pasar de -157,15 a -431,08 millones de pesos y se evidencia que entre los años 2010 y 2012 la tendencia es positiva mejorando año a año alcanzando para el año 2012 la cifra de $-138,83$ millones de pesos. La relación EVA/ANO mostró una evidente recuperación entre los años 2009 y 2012 al pasar de $-0,11$ a - $-0,01$ centavos por peso invertido en activos.

La caída de la participación de sector manufacturero en el PIB del Departamento Norte de Santander mostró la misma tendencia que se observó a nivel nacional donde el sector industrial disminuyó su aporte. Este comportamiento se mantuvo en el año 2013 donde la economía creció alrededor del 4,3\% y el sector industrial manufacturero presentó un decrecimiento de $1,2 \%$ a nivel nacional [6]. Esta situación podría evidenciar que los productos están perdiendo terreno frente a los foráneos con el correspondiente impacto en las cifras desempleo y contribución a la economía de la región. 
No. 2

Julio - Diciembre 2015 ISSN 0122-820X E-ISSN 2422-5053 PP: 54-72
En la investigación no se ubicó suficiente información financiera en la base de datos de Super sociedades de la clase 159 (Elaboración de bebidas) por lo cual fue descartada del estudio.

Al comparar los resultados del EVA del sector industrial manufacturero de la ciudad de Cúcuta con los resultados de otros estudios efectuados a nivel nacional [12], [13], [15], [20] se encontró que las empresas están destruyendo valor.

Se verifica que a excepción del año 2009 cuando el ROE estuvo por encima del CPPC durante el periodo de estudio el CPPC estuvo por encima del ROA y el ROE, esta situación de acuerdo a lo expresado por [15, p.9] explica la destrucción de valor en el sector ya que se reportaron utilidades por debajo del costo de capital.

En la Tabla VI se muestra el comportamiento del ROA, ROE y EVA en el sector industrial manufacturero de la ciudad de Cúcuta.

Tabla VI. ROA, ROE Y EVA en el sector industrial manufacturero de la ciudad de Cúcuta

\begin{tabular}{|l|c|c|c|c|c|}
\hline & 2008 & 2009 & 2010 & 2011 & 2012 \\
\hline ROA & 11,42 & 6,98 & 371 & 5,28 & 2,17 \\
\hline ROE & 11,39 & 11,39 & 6,06 & 8,95 & 4,59 \\
\hline CPPC & 13,20 & 10,05 & 9,09 & 9,47 & 9,77 \\
\hline EVA & -51.413 .65 & -27.635 .26 & -35.478 .98 & -38.699 .87 & -37.612 .12 \\
\hline
\end{tabular}

Fuente: Los Autores

Los resultados del EVA en el sector industrial manufacturero también se pueden atribuir a: desmejora en la rotación de activos y en la rotación de inventarios así como por la disminución del margen operacional.

En cuanto a la clase industrial minerales no metálicos, el análisis evidencia que se pueden atribuir los resultados a: disminución de la UODI por el incremento de los gastos de venta, al aumento del capital de trabajo neto operativo (KTNO) debido al crecimiento de las cuentas por cobrar (CxC) y los inventarios, otro factor es la disminución del margen operacional. Se verificó que el ROA y el ROE fueron menores que el CPPC en todo el período.

La clase industrial fabricación de muebles pudo verse afectada por la disminución de la rotación de activos así como la rotación de inventarios lo cual junto al crecimiento de las $\mathrm{CxC}$ incremento el valor del KTNO y por ende del activo neto de operación, se observó también el incremento de los activos fijos operacionales. Solo en el año 2010 el ROE fue mayor al CPPC en los demás años fue menor el ROA y el ROE.

Al revisar los resultados de la clase industrial fabricación de calzado, se evidencia que se pudo ver afectada también por la disminución de la rotación de activos, la reducción de la rotación de inventarios lo que incrementó el KTNO afectando los activos netos de operación. También se determinó que desmejoró la UODI influido el aumento de los gastos de ventas. En todos los años el ROA y el ROE estuvieron por debajo del CPPC.

El subsector productos de molinería, fabricación de alimentos para animales evidenció desmejora la rotación de activos y el margen operacional. A esto se suma la caída de la UODI por el incremento de los gastos operacionales de venta y al aumento de los activos netos de operación por la crecida del KTNO ocasionada por el aumento de las CxC. En todos los años el ROA y el ROE fueron menores al CPPC.

Aunque en la investigación se planteó un análisis cuantitativo con base en información contable, al contrastar con lo planteado por López donde señala que hay autores que indican que adoptar un pensamiento orientado a crear valor e identificar los elementos claves, lleva a la empresa sólo hasta la mitad del camino. Se plantea entonces a las empresas del sector en estudio adelantar una revisión de los procesos administrativos tal como se expone en [17, p.84 ] Este tópico está fuera del alcance de la presente investigación y se deja la inquietud como base para futuros trabajos. 


\section{Conclusiones}

Del estudio de la Creación de Valor mediante el indicador EVA en el sector industrial manufacturero de la ciudad de Cúcuta Departamento Norte de Santander Colombia en los años 2008 a 2012 se puede concluir:

- Enlaciudadexistepocavocaciónindustrial, el sector industrial manufacturero en el PIB ha venido perdiendo participación año a año lo que afecta a la economía de la región en sus indicadores sociales (generación de empleo) y en los económico por la caída del consumo intermedio y en consecuencia las variables inducidas en los demás sectores.

- El peso del sector en la economía nacional es muy bajo solo representa el $0,38 \%$ de la producción bruta.

- Dentro del sector industrial manufacturero de la ciudad de Cúcuta destacan en ese orden por su participación en el nivel de empleo, producción bruta $\mathrm{y}$ valor agregado a la economía las clases industriales: 269 Fabricación de productos minerales no metálicos, 369 Otras industrias manufactureras fabricación de muebles, 159 Elaboración de bebidas, 154 Productos molinería, almidones y alimentos para animales, 192 Fabricación de calzado.

- En la base de datos de Supersociedades no existe suficiente información para analizar la clase industrial 159 por lo que se descartó del análisis. Está fuera del alcance de este trabajo indagar las causas.

- El sector industrial manufacturero de la ciudad de Cúcuta de acuerdo al indicador EVA destruyó valor en todos los años del periodo de estudio. Se verificó que igual presentaron cada una de las clases industriales evaluadas. Se encontró que solo en el año 2009 la clase industrial otras manufacturas-fabricación de muebles presentó un valor positivo del EVA.
- Los resultados coinciden con otros estudios efectuados a nivel nacional en que las empresas del sector industrial manufacturero están destruyendo valor.

- Al analizar por tendencias generales se observó en el sector industrial manufacturero una tendencia hacia la mejora del indicador.

- En la clase industrial 269 la tendencia es negativa al igual que la clase industrial 154, mientras que la tendencia general de la clase industrial 369 es positiva así como la clase industrial 192.

- En todo el periodo de estudio el CPPC fue superior al ROA y al ROE lo cual de acuerdo la teoría del EVA implica destrucción de valor.

- El indicador EVA/ANO (centavos por peso invertido en activos) presenta una tendencia general positiva en el sector industrial manufacturero y se verifica que permaneció estable con pocas variaciones en los tres últimos años de estudio mostrando un valor de $-0,06$.

- Se evidencia en las clases industriales estudiadas que los indicadores financieros operacionales deben ser objeto de análisis ya que se detectaron valores y tendencias que indican que deben ser objeto de intervención y mejora.

- Al evaluar el contexto económico regional tomando como referencia la contribución al PIB departamental del PIB del sector estudiado contra los resultados del EVA en el período analizado, se evidenció que la variación del valor agregado de la industria manufacturera no presentó la misma tendencia que el PIB del sector. Mientras que la participación del PIB decreció año a año en ese periodo, el EVA por su parte presentó dos comportamientos muy definidos, entre los años 2008 y 2010 el indicador mostró tendencia a profundizar la destrucción de valor. A partir del
Julio - Diciembre 2015 ISSN 0122-820X E-ISSN 2422-5053 PP: 54-72 
No. 2 año 2010 el indicador EVA cambió la tendencia y empezó a mejorar aunque siguió presentando valores negativos es, decir el sector continuó destruyendo valor pero con tendencia a la mejora.

- Como resultado de la investigación se puede concluir que no se cumplió la hipótesis de trabajo "Las empresas del sector industrial manufacturero de Cúcuta en el periodo 2008-2012 crearon valor".

\section{Referencias}

[1] Ó.L. García. Valoración de empresas, gerencia de valor y EVA. Cali. Colombia. Prensa Moderna. 2003, pp. 129-248.

[2] TheWorldFactbook- CIA, Abril 2014. [Online]. Disponible en: https://www. cia.gov/library/.../the-world-factbook/.

[3] O. Amat. EVA Valor Añadido Económico: Un Nuevo Enfoque para Optimizar la Gestión Motivar y Crear Valor. España: Ediciones Gestión 2000. 2002, p. 32.

[4] I. Vélez. La creación de valor y su medida. Un análisis crítico de EVA. Ponencia. Bogotá, mayo 2000. [Online]. Disponible en: www.javeriana.edu.co/ decisiones/ponenciaeva.pdf.

[5] Informe sobre el desarrollo industrial. 2013. [Online]. Disponible en: http:// www.unido.org/fileadmin/user_media/ Research_and_Statistics/UNIDO_ IDR13_Spanish_overview_1118_for_ web.pdf.

[6] DANE. Boletines de prensa. 2013. [Online]. Disponible en https://www. dane.gov.co/files/investigaciones/ boletines/mtmr/bol_mtmr_IVtrim 12 . pdf.
[7] Caracol Radio. Crece preocupación por desempleo en Cúcuta. 2012, Marzo 01. [Online]. Disponible en: http:// caracol.com.co/radio/2012/03/01/ economia/1330594740_642623.html.

[8] F. Li Bonilla, "El Valor Económico Agregado(EVA) en el valor del negocio", Revista Nacional de Administración, vol. 1, no. 1, pp. 55-70, 2010. [Online]. Disponible en: http://unpan1.un.org/ intradoc/groups/public/documents/icap/ unpan044043.pdf.

[9] A. Rappaport. La creación de valor para el accionista. Buenos Aires: Deusto.260 p. 2006, p.28.

[10] L. Gitman. Principios de Administración Financiera. (14 Ed.) México: PearsonPrentice Hall. 2007, p.417.

[11] J. Rivera. Gestión basada en el valor. Cali: Programa Editorial de la Universidad del Valle. 2011, p.35.

[12] J. Rivera y A. Padilla, “Los medios de comunicación en Colombia son una industria creadora de valor?", Revista Finanzas y Política Económica, vol. 5, no. 2, pp. 89-113, 2013. [Online]. Disponible en: http://www.redalyc.org/ articulo.oa? $\mathrm{id}=323529880006$.

[13] J. Rivera y D. Ruíz, "Análisis del desempeño financiero de empresas innovadoras del sector alimentos y bebidas en Colombia", Pensamiento y gestión, no. 31, pp. 109-136, 2011. [Online]. Disponible en: http://rcientificas.uninorte.edu.co/ index.php/pensamiento/article/ view/3661/2378::pdf. 
[14] F. Rubio. Capital Asset Pricing Model (CAPM) y Arbitrage Pricing Theory (APT): una nota técnica, Finance, EconWPA. 2004. [Online]. Disponible en http://econpapers.repec.org/paper/ wpawuwpfi/0402007.htm.

[15] J. Rivera y D. Alarcón, “El cargo de Capital en la evaluación del desempeño financiero de empresas innovadoras de confecciones de Cali", Estudios gerenciales, vol. 28, no. 123, pp. 85-100. 2012. [Online]. Disponible en: http:// www.icesi.edu.co/revistas/index.php/ estudios_gerenciales/article/view/1209/ html.

[16] U. Garay y M. González. Fundamentos de Finanzas. Caracas,Venezuela: Ediciones IESA, 2007, p. 290.

[17] D. R. Gómez. Una herramienta para la toma de decisiones gerenciales: la creación de valor (EVA). [Online]. Disponible en: http://www.ugr. es/ rgomezl/documentos/publiclibros/ CreacionValor/CreaciondeValor.pdf.

[18] D. Alfaro, J. Segura y N. Gutiérrez, "Análisis de Creación de Valor Económico para Empresas del Sector Textil (Valueaddedanalysisforthetextile sector businesses)", Tec Empresarial, vol. 5, no.1, pp. 41-48, 2011. [Online]. Disponible en: http://www.tec-digital. itcr.ac.cr/servicios/ojs/index.php/tec_ empresarial/article/view/610.

[19] S. Radi y S. Bolívar, "Creación de Valor de las Empresas Colombianas Durante el Período 2000-2005", Pensamiento y Gestión, no. 22, 2011. [Online]. Disponible en: http://rcientificas.uninorte.edu.co/ index.php/pensamiento/article/ view/3529/2259::pdf.
[20] G. Escobar, R. Arango, A. Molina y F. Arias, (Diciembre 2011). Medición del valor económico agregado EVA de las empresas de Caldas en el periodo 20002008. Lúmina 12, Enero - Diciembre, 2011. págs. 196 - 215. [Online]. Disponible en http://www.umanizales. edu.co/publicaciones/campos/ economicas/lumina/recursos/12/5 medicion.pdf.

[21] G. Escobar, "Las empresas de alimentos de Manizales generan ganancias pero destruyen valor económico agregado (EVA)", Ánfora, año 15, no. 25, 2008. [Online]. Disponible en: http://www.redalyc.org/articulo. oa? $\mathrm{id}=357834257015$.

[22] SIREM -Superintendencia de Sociedades. Sistema de información y de riesgo empresarial-SIREM. 2013. [Online]. Disponible en: http://sirem. supersociedades.gov.co/Sirem2/index. jsp.

[23] Encuesta Anual Manufacturera -EAMDANE. 2014. [Online]. Disponible en: http://www.dane.gov. co/index.php/construccion-enindustria/industria/encuesta-anualmanufacturera-eam.

[24] Cámara de Comercio de Cúcuta. 2013. PIB. [Online]. Disponible en: http:// www.datacucuta.com/\#! pib/cxt7.

ISSN 0122-820X

ISSN 2422-5053

P: 54-72

[25] Damodaran Online: Home Page for Aswath Damodaran. 2014. [Online]. Disponible en: www.stern.nyu. edu/ adamodar/.

[26] Banco Mundial. Indicador. Marzo 2014. [Online]. Disponible en: http://datos. bancomundial.org/indicador/FR.INR. LEND/countries. 
No. 2

Julio - Diciembre 2015 ISSN 0122-820X

E-ISSN 2422-5053 PP: $54-72$

[27] Banco Central de Perú. (Marzo 2014). Estadísticas. [Online]. Disponible en: http://www.bcrp.gob.pe/docs/ Estadisticas/Cuadros-Estadisticos/ NC_037.xls.

[28] Portal Ambito.com. Riesgo País. Marzo 2014. [Online]. Disponible en: http:// www.ambito.com/economia/mercados/ riesgo-pais/info/? id=4.

[29] México Máxico. Tabla de inflación anual en países. Marzo 2014. [Online]. Disponible en: www.mexicomaxico. org/Voto/Inflacion.htm. 